\section{The Macrophage-Exploiting Hypoxia With Cell Based Delivery of GDEPT}

\author{
S.M. Kingsman, L. Griffiths, K. Binley, S. Iqball, \\ O. Kan, P. Maxwell, C. Lewis, A. Harris \& S. \\ Naylor
}

\title{
A Physiologically Regulated Adenoviral Vector for the Treatment of Ischaemic Disease
}

\author{
S.M. Kingsman, K. Binley, L. Griffiths, S. \\ Iqball, H. Spearman, A.J. Kingsman \& S. \\ Naylor
}

Oxford Biomedica (UK) Ltd, Medawar Centre, Oxford Science Park, Oxford, OX4 4GA, UK

Adenoviral vectors can be used for the transient expression of a variety of genes in both dividing and non-dividing cells. Although such a broad tropism can be advantageous there is the possibility that non-target cells might be adversely affected by the expression of a transferred gene. To overcome this limitation we have developed an adenoviral vector that can deliver regulated gene expression in response to physiological stimuli. A large number of pathological conditions are characterised by ischaemia where oxygen and glucose levels are low and the tissues are acidic. These conditions activate a number of genes and in particular low oxygen (hypoxia) is sensed by DNA sequence elements referred to as hypoxia response elements. We have characterised a number of such elements and configured them into adenoviral vectors. In one configuration we have obtained a vector which displays a remarkable degree of regulation in response to hypoxia, basal expression is minimal and maximum expression is equivalent to that obtained with commonly used constitutive promoters. We have characterised the expression of this vector in a range of target tissues including human tumour cells, xenografts and normal tissues including skeletal muscle and hematopoeitic cells. This vector can be used to deliver therapeutic genes such as pro-drug activating enzymes and vascular growth factors for the treatment of diseases such as cancer, peripheral arterial disease and arthritis. 